Onkologie 1995;18:2-4

\title{
Contents, Vol. 18, No. 1, 1995
}

Inhalt

\section{Editorial}

ONKOLOGIE 1995: The Completed Editorial Staff Realizes

New Concepts

Queißer, W., Huber, H

Editorial

ONKOLOGIE 1995: Eine ergänzte Schriftleitung verwirklicht neue Konzepte

Queißer, W., Huber, $\mathrm{H}$

\section{Short Reviews}

Current Primary Surgery of Thyroid Carcinoma

Gimm, O., Dralle, H

Kurze Übersichten

Gegenwärtiger Stand der chirurgischen Primärtherapie des Schilddrüsenkarzinoms

Gimm, O., Dralle, $\mathrm{H}$

Male Breast Cancer

Schön,M., Zaiac, M., Schlag,P.M 16

Das Mammakarzinom des Mannes

Schön, M, Zaiac, M., Schlag, P. M. .

16

Original Articles

Prevention of Cisplatinum-Induced Delayed Emesis and Nausea

Münstedt, K., Milch, W., Blauth-Eckmeyer, E., Spänle, A.,

Vahrson, H., Reimer, Ch 23

4'-Iodo-4'-Deoxydoxorubicin (IDX) in Colorectal Cancer -A Multicenter Phase II Study

Herrmann, R., Mross, K., Kleeberg, U., Kreuser, E. D., Becher, R.,

Edler, L., Queißer, W., Liati, P., Kerpel-Fronius, S. for the Phase II

Study Group of the Association of Medical Oncology of the

German Cancer Society 28

De Novo Malignancies Following Renal Transplantation: Experience of a Single Center

Bürstner, O., Marchner, M., Land, W.

Originalarbeiten

Vorbeugende Therapie bei Cisplatin-induzierter, verzögerter Ubelkeit und Erbrechen

Münstedt, K., Milch, W., Blauth-Eckmeyer, E., Spänle, A.,

Vahrson, H., Reimer, Ch 
4'-Iodo-4'-Deoxydoxorubicin (IDX) beim kolorektalen Karzinom -Eine multizentrische PhaseII-Studie

Herrmann, R., Mross, K., Kleeberg, U., Kreuser, E. D., Becher, R.,

Edler, L., Queißer, W., Liati, P., Kerpel-Fronius, S. für die

Phase-II-Studiengruppe der Arbeitsgemeinschaft für Internistische

Onkologie der Deutschen Krebsgesellschaft 28

De-novo-Malignome nach Nierentransplantation: Ergebnisse am Klinikum Großhadern

Bürstner, O., Marchner, M., Land, W.

32

Ongoing Clinical Trials

2-Chlorodesoxyadenosine: First-Line Treatment for Low-Grade Non-Hodgkin's Lymphomas. A Phase II/III Study of the 'Arbeitsgemeinschaft Medikamentöse Tumortherapie'

Fridrik, M. A., Felbermayr, M., Linkesch, W., Neubauer, M.,

Schiller, L., Hausmaninger, H., Oppitz, P., Gattringer, C

i

Autologous Transplantation in Patients with Acute Myeloid Leukemia in First Remission with IL-2-Cultured Marrow or Peripheral Blood Stem Cells Followed by in vivo IL-2

Klingemann, H.-G, Eaves, C. L, Barnett, M. J., Eaves, A. C,

Hogge, D. E., Lansdorp, P., Nantel, S. H, Reece, D. E., Shepherd, J. D.,

Sutherland, H.J., Phillips, GL 44

Effectiveness and Utility of a Second-Line Treatment in Metastatic Breast Cancer

Porzsolt, E, Mayer-Steinacker, R., Eggl, C 48

Laufende Klinische Studien

2-Chlordesoxyadenosin in der Primärtherapie niedrigmaligner Non-Hodgkin-Lymphome. Eine Phase-II/III-Studie der «Arbeits-gemeinschaft Medikamentöse Tumortherapie»

Fridrik, M. A., Felbermayr, M., Linkesch, W., Neubauer, M.,

Schiller, L., Hausmaninger, H, Oppitz, P., Gattringer, C 39

Autologe Transplantation von IL-2-kultiviertem Knochenmark oder peripheren Stammzellen mit

IL-2-Nachbehandlung bei Patienten mit akuter myeloischer Leukämie in erster Remission

Klingemann, H.-G, Eaves, C. J., Barnett, M. J., Eaves, A. G,

Hogge, D. E., Lansdorp, P., Nantel, S. H., Reece, D. E., Shepherd, J. D.,

Sutherland, H.J., Phillips, GL 44

Wirksamkeit und Nutzen einer Salvage-Therapie bei Patientinnen mit metastasierendem

Mammakarzinom

Porzsolt, E, Mayer-Steinacker, R., Eggl, C 48

Casuistic Contributions

Hepatic Injury Due to G-CSF Application

Büntzel, J., Küttner, K

54

Kasuistiken

Hepatitisches Versagen nach G-CSF-Gabe

Büntzel, J., Küttner, K

54

Continued on page 4 
Fortsetzung aufSeite 4

Band 18, Heft 1, Februar 1995

International Journal for Cancer Research and Treatment

Contents

Inhalt

The Spontaneous Perforation Risk of the T-Cell Lymphoma of the Small Intestine

Kramer, W., Richter, H. J., Hoffmeister, N., Werner, H.|P. 58

Zum spontanen Perforationsrisiko des T-Zell-Lymphoms des Dünndarms

Kramer, W., Richter, H. J., Hoffmeister, N., Werner, H. P..

58

Letter to the Editors

Thürlimann, B., concerning:

Vijayakumar S, et al: Localized prostate cancer:

Use of serial prostate-specific antigen measurements during

radiation therapy - An update. Onkologie

1994;17:578-585

63

Brief an die Herausgeber

Thürlimann, B., zu:

Vijayakumar S, et al: Lokalisiertes Prostatakarzinom:

Der Nutzen der serienmäßigen Bestimmung des prostata-spezifischen

Antigens während der Strahlentherapie - Ein Update. Onkologie

1994;17:578-585

63

Commentary

The Role of Folinic Acid in Combination with 5-Fluorouracil in the Treatment of Advanced

Colorectal Cancer

Wilke, H., Harstrick, A., Seeber, S

65

Kommentar

Die Rolle von Folinsäure in Kombination mit 5-Fluorouracil bei der Behandlung des fortgeschrittenen kolorektalen Karzinoms

Wilke, H., Harstrick, A., Seeber S 65

Oncological Education

Alternative Strategies in Medical Tumor Therapy

Nagel, A

Therapeutic Strategies in Malignant Lymphomas

Engelhard, M., Pfreundschuh, M., Engert, A., Diehl, V., Goldstone, A. H., Freund, M., Schmitz, N., Hiddemann, W.

Onkologische Fortbildung

Alternative Strategien in der medikamentösen Tumortherapie

68 Nagel, A 
Therapiestrategien bei malignen Lymphomen

Engelhard, M., Pfreundschuh, M., Engert, A., Diehl, V., 75 Goldstone, A. H., Freund, M., Schmitz, N., Hiddemann, W. .

75

Clinical Information

Notes on the Planning and Implementation of Clinical Trials in the Fields of Somatic Cell and Gene Therapy

Lindemann, A., Rosenthal, F. M., Hase, St., Markmeyer, P.,

Mertelsmann, $\mathrm{R}$ 85

Klinische Information

Hinweise zur Planung und Durchführung klinischer Studien in den Bereichen somatische Zellund Gentherapie

Lindemann, A., Rosenthal, F. M., Hase, St., Markmeyer, P.,

Mertelsmann, $\mathrm{R}$

85

Congress Reports 90 Kongreßberichte

90

Wilsede School for Oncology and Hematology

92 Wilsede-Schule für Onkologie und Hämatologie ... 92

Book Review

Buchbesprechung

“

Reports of Oncological Societies loo Mitteilungen onkologischer Gesellschaften

100

Imprint 1 Impressum 1

Instructions to Authors 103 Hinweise für Autoren 103

Bibliographischer Hinweis: Inhaltsverzeichnisse dieser Zeitschrift erscheinen regelmäßig in current contents ${ }^{\circledR}$ sowie in anderen bibliographischen Diensten. 